\title{
Crédit de trésorerie des producteurs de coton au centre du Bénin : modalités et conséquences
}

\author{
Koffi Benoît Sossou ${ }^{1}$ et Michel Fok ${ }^{2,3, *}$ \\ ${ }^{1}$ Centre de formation et de recherche en matière de population, Université Abomey-Calavi, Bénin, France \\ 2 CIRAD, UPR AIDA, 34398 Montpellier, France \\ 3 AIDA, Univ Montpellier, CIRAD, Montpellier, France
}

\begin{abstract}
Résumé - Alors que les besoins de trésorerie sont accrus par la nécessité de rémunérer la main-d'œuvre pour compléter le travail fourni par la famille, l'octroi de crédit de trésorerie est peu pris en compte dans l'organisation de la production cotonnière en Afrique francophone. Cette étude est la première à aborder les conditions d'octroi et les impacts du crédit informel de trésorerie pour les producteurs de coton. L'étude est basée sur l'analyse d'une politique particulière menée au Bénin, en 2012, d'octroi formel de crédit de trésorerie aux producteurs de coton. L'étude concerne le centre du Bénin où la politique mise en œuvre de crédit formel a aussi attiré les opérateurs de crédit informel qui ont ainsi révélé les conditions de leur intervention. Avec la collaboration de 537 producteurs de coton relevant de 15 coopératives, l'étude montre que très peu d'entre eux ont échappé au recours aux opérateurs informels pour obtenir de la trésorerie, avec application d'un taux d'intérêt annuel pouvant dépasser $200 \%$. En valeur, le remboursement de ce crédit, utilisé pour diverses fins difficiles à suivre précisément, peut équivaloir, voire dépasser celui du crédit acquis pour les intrants, absorbant ainsi une grande partie de la marge monétaire dégagée par la culture du coton. Les conditions observées du crédit informel ont enfermé les producteurs dans une trappe d'endettement et de pauvreté, au bénéfice d'acteurs ignorés de la filière cotonnière. Une absence ou une inadaptation de politique de crédit formel de trésorerie aux paysans cultivant du coton peuvent ainsi transférer l'essentiel du profit de cette culture aux acteurs de crédit informel et usuraire. Le recours au crédit de trésorerie devrait être davantage étudié et pris en compte dans l'organisation et la gestion des filières cotonnières.
\end{abstract}

Mots clés : crédit à court terme / trésorerie / taux d'intérêt / coût de production / commercialisation / Bénin

Abstract - Cash credit to cotton producers in Central Benin: Modalities and implications. While the requirement for cash has been increasing along the need to pay labor external to family, the provision of cash credit is little taken into account in the management of cotton sectors in French speaking African countries. Our study is the first one to address the conditions and impacts of the informal provision of cash credit to cotton producers in that region. The study is based on the appraisal of a particular policy implemented in Benin, in 2012, to formally provide cash credit to cotton producers. It is focused on the center of Benin where the implementation of the policy has attracted informal operators to join in, hence revealing the conditions on how they operated. Thanks to the collaboration of 537 cotton producers involved in 15 cooperatives, our study showed that very few farmers have escaped from the intervention of informal players operating at interest rate that could exceed $200 \%$ per year. The amount to reimburse cash credit, whose various destined usages were not monitored, could reach if not exceed that for input credit, hence almost swallowing the monetary margin from cotton growing. The observed conditions of the cash credit by informal operators were capturing farmers into a trap of debt and poverty, to the benefit of hidden players and ignored by the cotton sector organization. The lack or the inaccuracy of formal provision of cash credit to cotton producers could mainly lead to pass the main benefit of cotton cultivation to players of informal and usury credit. The recourse to cash credit by cotton producers should be further analyzed and integrated into the organization and management of cotton sectors in African countries.

Keywords: short term credit / cash credit / interest rates / production cost / marketing / Benin

\footnotetext{
$\overline{\text { *Auteur de correspondance }}$ : michel.fok@cirad.fr
} 


\section{Introduction}

Le caractère de trappe de pauvreté du crédit informel parait s'imposer avec la tragédie de plusieurs épisodes de suicide de paysans en Inde (Sadanandan, 2014), ces derniers ayant préféré l'issue fatale plutôt que de se voir dépossédés de leurs terres qu'ils avaient gagées pour obtenir des crédits auprès des usuriers (Posani, 2009). Cette tragédie a particulièrement touché les zones cotonnières du pays, au point que de nombreux observateurs en ont rendu responsable soit la culture de coton, soit le recours aux variétés transgéniques, même si les premiers épisodes de suicide étaient antérieurs à l'utilisation de ces variétés (Visvanathan, 1998).

Il n'y a cependant pas de fatalité à la trappe de pauvreté associée au recours au crédit, comme en témoignent les appréciations longtemps positives sur les filières cotonnières dans les pays d'Afrique francophone. Le succès de ces filières était jugé fondé notamment sur l'organisation de la fourniture des intrants à crédit de manière formelle (Lele et al., 1989), ce que des analyses plus récentes ont réaffirmé (Poulton et al., 2004; Thériault et Tschirley, 2014).

En Afrique, l'accent mis sur l'accès aux intrants à crédit pour produire a fait négliger l'analyse des crédits de trésorerie, surtout dans les pays cotonniers de l'Afrique francophone. En Asie, les modalités d'usure des crédits informels, avec des taux d'intérêt approchant les $100 \%$, ont été observées au Népal (Adams et al., 2003), mais ces crédits ne toucheraient qu'une partie des paysans, tout comme au Myanmar (Kaino, 2006). En Afrique, Defo (2005) a indiqué que les commerçants n'étaient pas les seuls à prêter de l'argent aux paysans. Le banquier ambulant ou garde-monnaie - observé au Bénin, Togo et au Cameroun, mais aussi au Ghana (Quartey et al., 2012), qui collecte l'argent des paysans, le garde et le restitue au bout d'une période, moins une rémunération pour service rendu - peut aussi prêter de l'argent dans des conditions moins bien connues que pour les commerçants. Ces derniers consentent des crédits de courte durée aux personnes en situation de besoin urgent de liquidité (Defo, 2005) à des taux d'intérêt mensuel de 5 à $20 \%$ par mois (soit 60-240\% par an). De tels taux élevés sont pratiqués aussi par les associations traditionnelles d'épargne et de crédit dans les zones rurales du Nigeria, mais seulement à l'endroit des emprunteurs qui n'en sont pas membres (Eboh, 2000).

Le présent article vise à cerner l'étendue, les modalités et l'incidence du recours au crédit informel de trésorerie sur la rentabilité de la culture cotonnière dans le cas du Bénin et dans un contexte particulier avec la mise en œuvre, en 2012, d'une mesure gouvernementale pour octroyer du crédit de trésorerie de manière formelle, mais sans réussir à supprimer le crédit informel, bien au contraire.

\section{Matériel et méthodes}

\subsection{Présentation du cadre d'étude}

L'étude a été réalisée dans le département des Collines au centre du Bénin (Fig. 1) où la production cotonnière a baissé depuis une dizaine d'années (Kpadé, 2011).

\subsection{Contexte, données et leur traitement}

L'étude a été réalisée en 2012 dans un contexte particulier où la mesure gouvernementale d'octroi de crédit de trésorerie a été mise en œuvre par deux organismes formels et où des opérateurs informels se sont engouffrés également.

Les matériaux mobilisés pour l'étude conduite concernent la campagne agricole 2012-2013. Ils proviennent des cahiers de tenue des crédits au niveau des Coopératives villageoises de producteurs de coton (CVPC) et des très nombreux entretiens avec les paysans dans les villages. L'étude a concerné trois des six Communes du département des Collines (Savé, Glazoué et Dassa), et a été réalisée dans 15 CVPC sur un total de 102. Les 15 CVPC ont été choisies pour représenter trois types de localités à dominante d'autochtones (à Glazoué), d'allochtones d'origine proche (Dassa), et d'origine plus lointaine (Savé). Tous les producteurs de coton des 15 CVPC ont été intégrés dans l'étude, soit un nombre total de 537.

Les longs entretiens avec les producteurs des CVPC étudiées, combinés à l'accès aux cahiers de crédit tenus par les CVPC, ont permis de comprendre les modalités d'octroi et de remboursement des crédits de trésorerie, et de reconstituer les données de crédit pour tous les producteurs de l'étude, aussi bien pour les intrants obtenus auprès des sociétés cotonnières que pour les crédits de trésorerie. Ce gros travail de reconstitution des données a été complété par une enquête en un passage pour relever les éléments structurels relatifs aux chefs d'exploitations (âge, niveau d'éducation...), aux familles concernées (nombre d'épouses, nombre de jeunes participants aux travaux dans les champs...) et aux exploitations (surface totale cultivée, pratique de la culture attelée).

L'exploitation du contenu des cahiers des CVPC a été facile pour les crédits intrants, mais beaucoup moins pour le crédit de trésorerie. Pour ce dernier, il a fallu distinguer les crédits alloués par les deux opérateurs formels (cf. infra) et par les opérateurs informels. Dans le cas des opérateurs informels, le crédit octroyé à chaque paysan était indiqué seulement par la date d'obtention du crédit, le montant à rembourser et la date de remboursement effectif. Il a fallu s'entretenir avec chaque paysan, évoquer les reconnaissances de dettes qu'ils avaient signées, pour reconstituer les montants réels des crédits obtenus auprès des opérateurs informels. Cette reconstitution détaillée était nécessaire pour en déduire les taux d'intérêt effectivement appliqués par les prêteurs informels.

Les données collectées n'ont pas permis de cerner l'incidence du crédit de trésorerie sur les coûts de production du coton, mais seulement sur la rentabilité de cette culture. Les producteurs en contrainte de liquidité le sont soit pour acquérir des facteurs de production, comme la main-d'œuvre temporaire, soit pour faire face à des dépenses sociales, voire pour les deux raisons à la fois, mais les entretiens avec les producteurs n'ont pas permis d'identifier de manière correcte les destinations des crédits obtenus. Par contre, les crédits sont forcément à rembourser sur le revenu de la culture cotonnière, l'acquisition de crédit de trésorerie impacte mécaniquement la rentabilité de cette culture.

Pour appréhender l'incidence sur la rentabilité, le ratio classique extrant/intrant - une valeur de 3 du rapport de la valeur de la production sur le coût des intrants est souvent prise comme seuil de rentabilité d'une intensification par les intrants - est complété par deux indicateurs de marge financière et la distribution des producteurs selon des classes de marge financière. Il s'agit d'abord de la marge après remboursement des intrants (MARI) couramment utilisée dans les études portant sur la production cotonnière en Afrique 


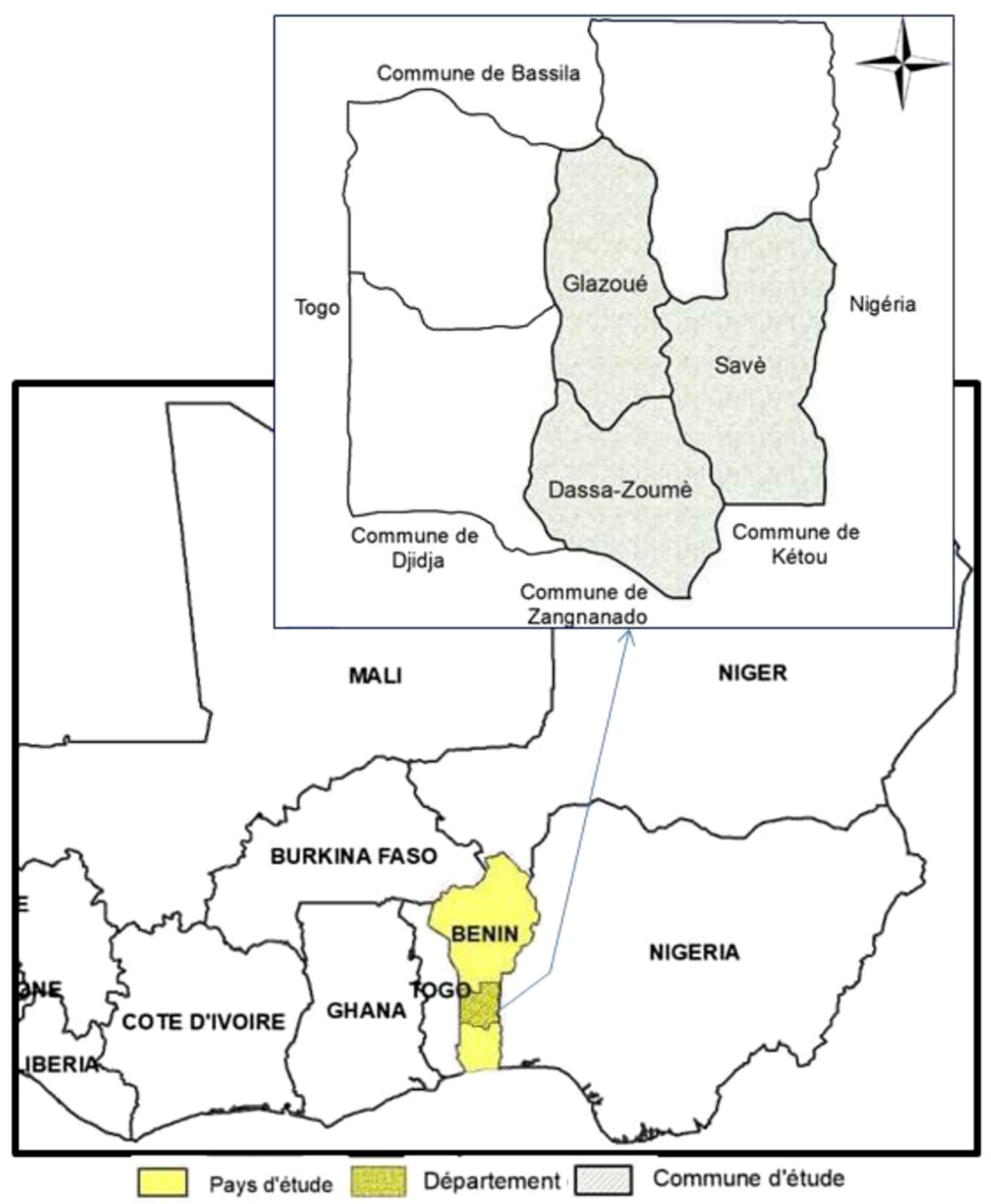

Fig. 1. Secteur d'étude : département des Collines au centre du Bénin.

Fig. 1. Study area: Province of Collines, at the center of Benin.

francophone. Pour aller au-delà du crédit sur les intrants, exclusivement considéré dans de nombreuses études sur le coton, nous avons introduit en supplément la marge après le remboursement des intrants et des crédits hors intrants (MARICHI), ces derniers correspondant aux crédits de trésorerie. Ces deux indicateurs sont libellés à l'hectare, car les crédits pour les intrants et la trésorerie sont censés être remboursés par la culture du coton. Quatre classes de rentabilité selon quatre valeurs de MARICHI, y compris la valeur négative, ont été identifiées pour cerner l'incidence du crédit de trésorerie sur les producteurs par leur répartition dans ces classes.

Après une analyse descriptive des résultats pour indiquer la fréquence du recours au crédit de trésorerie (formel et informel) de la campagne 2012-2013, les montants représentés, les taux d'intérêt appliqués et l'incidence sur la rentabilité de la culture cotonnière, les facteurs liés aux profils des producteurs et pouvant influencer la demande de crédit de trésorerie (notamment pour payer de la main-d'œuvre extrafamiliale) ont été testés par régressions logistiques. Les 
modèles choisis n'obéissent pas à une théorie spécifique de recours au crédit de trésorerie. Pour expliquer le recours à plusieurs crédits au lieu d'un seul, les variables explicatives choisies et les signes des effets attendus (Tab. 4) découlent des connaissances sur les contraintes des exploitations agricoles.

\section{Résultats et discussion}

\subsection{Acteurs et modalités du crédit de trésorerie}

\subsubsection{Modalités des opérateurs formels}

L'engagement du crédit spécial dans le département des Collines a fait intervenir deux types d'organisation. L'organisation non gouvernementale CFAD (Centre pour la formation et l'appui au développement) a été spécifiquement retenue pour la mise en œuvre en plus des Caisses locales de crédit agricole mutuel (CLCAM). Ces dernières étaient opérationnelles depuis 1987 pour octroyer aux commerçants des crédits sur 10 mois avec un taux d'intérêt de $20 \%$ et une pénalité de retard de $10 \%$ par mois. Ce sont ces modalités qui se sont appliquées aux producteurs cotonniers, avec seulement un ajustement de la période d'octroi de crédit. L'ONG CFAD, opérateur principal, s'est alignée sur la même modalité en termes de durée du crédit (10 mois) avec cependant un taux d'intérêt moindre de $5 \%$ et sans application de la pénalité de retard.

L'accès aux crédits des CLCAM et du CFAD était très administratif, suivant des modalités peu adaptées au cas des paysans. Chaque producteur devait remplir et signer une fiche de demande de crédit spécifiant entre autres le chiffre d'affaires prévisionnel de la campagne. L'octroi du crédit demandé était conditionné notamment à l'avis motivé du comité de CVPC.

Les procédures mises en œuvre par les organismes formels induisaient de fait l'approbation des crédits de trésorerie par les CVPC, et donc leur adossement à la commercialisation du coton, mais sans implication des sociétés cotonnières. Au paiement du coton commercialisé, la somme versée aux producteurs était d'abord défalquée du prélèvement pour rembourser le crédit intrants aux sociétés cotonnières, puis de celui pour rembourser les crédits de trésorerie inscrits dans les cahiers de crédit tenus par les CVPC.

\subsubsection{Modalités des opérateurs informels}

L'intervention des opérateurs informels était motivée aussi bien par la nécessité des producteurs de se tourner vers eux que par l'intérêt ressenti par ces opérateurs d'y répondre. Les besoins des producteurs n'étaient pas couverts suffisamment par les opérateurs formels. Du côté des opérateurs de crédit informel, l'adossement déjà indiqué des crédits de trésorerie à la commercialisation du coton leur conférait une bonne sécurité de recouvrement.

La nature des opérateurs informels était très diverse, mais les conditions appliquées étaient les mêmes. Les commerçants n'étaient pas les seuls à intervenir, ils étaient complétés par des paysans un peu plus aisés et ayant des liquidités à prêter, des enseignants installés dans les zones rurales, des fonctionnaires ou leurs épouses, mais aussi des associations de parents d'élèves. C'est une diversité d'intervenants que Defo (2005) avait déjà soulignée. Tous ces intervenants se sont alignés sur des taux d'intérêt de $50 \%$ pour un remboursement dans les six mois suivant l'octroi du crédit, et de $100 \%$ au-delà, avec une limite de dix mois. Dans la réalité, le taux de $100 \%$ s'est appliqué de manière quasi-exclusive du fait du retard du paiement du coton par les sociétés cotonnières.

Le caractère usuraire du crédit informel pouvait être renforcé par l'obligation pour les emprunteurs de s'engager dans la vente par anticipation (ou achat sur pied), mais à vil prix, des autres productions monétaires des paysans. Ainsi, «le soja sur pied» était cédé au prix de $3000 \mathrm{~F} \mathrm{CFA/bassine} \mathrm{(de} 30$ à $32 \mathrm{~kg}$ ) alors que le prix observé après la récolte était de $9000 \mathrm{FCFA} /$ bassine $(1 €=656 \mathrm{FCFA})$. Le kilo de noix de cajou vendu à l'avance était cédé à $100 \mathrm{~F} \mathrm{CFA} \mathrm{contre} 300$ à $350 \mathrm{~F} \mathrm{CFA}$ à la période de la récolte. Ce sont des écarts de prix très nettement supérieurs à ceux observés au Ghana (Quartey et al., 2012).

\subsubsection{Coercition et trappe de pauvreté}

En dépit de l'adossement du crédit à la commercialisation du coton-graine, le remboursement du crédit informel pouvait ne pas être total pour diverses raisons (aléas climatiques, maladie du paysan...), donnant alors lieu à des mesures de coercition. Sur la base de la reconnaissance de dette produite par le prêteur (le plus souvent un commerçant), le paysan défaillant est convoqué à la gendarmerie, mis en détention, et le plus souvent libéré seulement après s'être engagé, par une nouvelle reconnaissance de dette, à rembourser le reliquat du prêt l'année suivante avec un taux d'intérêt additionnel de $100 \%$. À titre d'illustration, un paysan qui aurait eu à rembourser $100000 \mathrm{FCFA}$ pour avoir contracté un crédit de $50000 \mathrm{FCFA}$ et qui n'aurait remboursé que $60000 \mathrm{FCFA}$, aura à signer un nouvel engagement de $80000 \mathrm{FCFA}$ pour rembourser le reliquat de $40000 \mathrm{~F} \mathrm{CFA} \mathrm{la} \mathrm{campagne} \mathrm{suivante.}$ Sur deux campagnes, il aura ainsi payé $140000 \mathrm{~F}$ CFA pour avoir contracté une dette initiale de $50000 \mathrm{FCFA}$. La complicité des forces de l'ordre dans la mise en œuvre des mesures de coercition faisait peu de doute aux yeux des paysans interrogés.

La trappe de pauvreté était actionnée en cas de défaut dans le remboursement du crédit informel car un processus vicieux se trouvait enclenché. Le coût financier à assumer pour le remboursement du crédit de l'année N-1 n'affranchit en effet pas du besoin de s'engager dans un nouveau crédit pour produire du coton en année $\mathrm{N}$, seule possibilité pour le paysan d'espérer les rentrées monétaires indispensables pour espérer pouvoir s'acquitter de sa dette. Une telle possibilité, dans les conditions d'octroi du crédit décrites ici, pouvait s'avérer n'être qu'une illusion.

\section{2 Étendue, impacts et facteurs du crédit informel}

\subsubsection{Crédit largement répandu et informel}

Le nombre de crédits de trésorerie enregistrés dans les cahiers des CVPC a été supérieur au nombre de producteurs suivis dans l'étude $(171+492=663$ crédits pour 537 producteurs), indiquant que des producteurs avaient, en moyenne, eu recours à plus d'un crédit au cours de la campagne 2012-2013 (Tab. 1). Les producteurs ont fréquemment eu recours au crédit auprès des acteurs informels, même quand ils en avaient obtenus auprès des acteurs formels; un certain nombre d'entre 
Tableau 1. Caractéristiques des crédits de trésorerie.

Table 1. Cash credit characteristics.

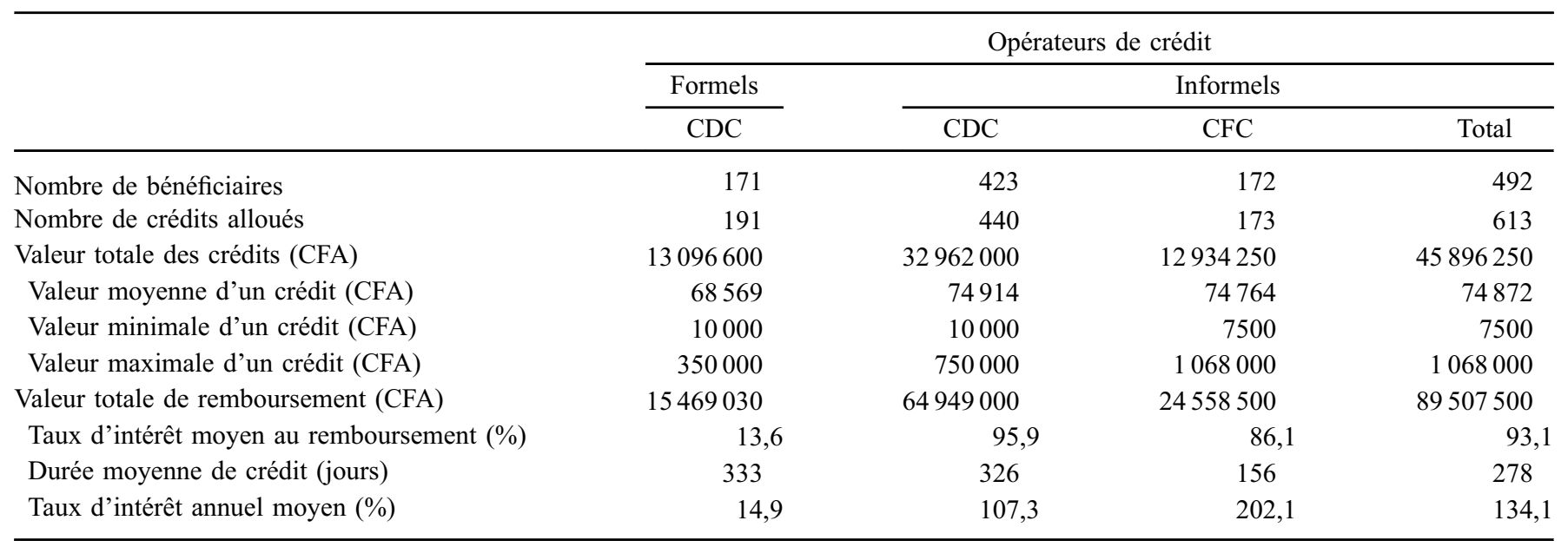

CDC : crédit de début de campagne (avant le 15 juillet); CFC : crédit de fin de campagne (après le 15 octobre); nombre de bénéficiaires : nombre de paysans ayant bénéficié de crédit formel ou informel, indépendamment du nombre de crédits de chaque type; une centaine de paysans recourant aux acteurs informels ont été bénéficiaires de CDC et de CFC, ce qui explique que le nombre de bénéficiaires de crédits informels, sans distinction de période, soit inférieur à la somme des nombres de bénéficiaires de CDC et de CFC.

eux ont recouru aux acteurs informels aussi bien en début de campagne qu'en fin de campagne.

Les crédits octroyés par les opérateurs informels ont représenté près des $3 / 4$ du total des crédits enregistrés en début et en fin de campagne, alors que les opérateurs formels n'ont fourni des crédits qu'en début de campagne.

En termes de valeur totale, le montant des crédits fournis par les acteurs informels a été plus de trois fois celui assuré par les deux entités formelles. Les montants moyens de crédit ont été similaires pour les deux types de crédit (formels et informels), mais les montants maximaux ont été plus élevés avec le crédit informel.

Au total, la valeur au remboursement du crédit informel a été bien supérieure à celle du crédit formel (dans un rapport de près de 6 pour 1), en conséquence d'un volume de crédit plus élevé et du taux d'intérêt largement supérieur. Le paiement tardif du coton pour la campagne considérée, vers avrilmai 2013, a contribué à faire dépasser la durée maximale de six mois fixée par les prêteurs pour pouvoir bénéficier d'un taux d'intérêt plus faible.

\subsubsection{Crédit usuraire des opérateurs informels}

Pour ce qui concerne le crédit alloué par les deux entités formelles, le taux moyen d'intérêt au remboursement a été de $13,6 \%$ - ce qui paraît raisonnable dans le contexte des pays africains - mais celui du crédit informel a été bien illustratif du crédit usuraire $(93,1 \%)$. Ce taux, très proche de $100 \%$, indique que rares ont été les producteurs capables de rembourser les opérateurs de crédit informel dans les six mois suivant l'obtention du crédit.

Le caractère usuraire a été encore plus flagrant en considérant la durée effective du crédit, inférieure à l'année, surtout s'agissant du crédit informel (278 jours contre 333 jours pour le crédit formel): rapporté à l'année, le taux d'intérêt moyen du crédit informel dépasse $100 \%(122,2 \%)$ et est très largement supérieur à celui du crédit formel $(14,9 \%)$.
Le caractère usuraire a surtout été extrême pour les crédits alloués en fin de campagne - exclusivement assurés par les opérateurs informels - et qui ont été remboursés à la même période que les crédits de début de saison agricole, après le paiement du coton. Rapportés à l'année, les taux d'intérêt réclamés par les acteurs informels ont été respectivement de 107,3 et $202,2 \%$, pour les crédits de début de campagne et pour ceux de fin de campagne. Ce sont des taux qui sont égaux ou supérieurs à ceux identifiés en Asie (Barslund et Tarp, 2008; Kaino, 2006), mais avec des écarts de taux entre les opérateurs formels et informels nettement plus élevés (Bell, 1990; Barslund et Tarp, 2008). Les niveaux que nous avons calculés permettent de comprendre pourquoi les microcrédits, distribués avec un taux médian d'intérêt d'environ $30 \%$ dans les pays au sud du Sahara (Rosenberg et al., 2009), ont été perçus positivement par les bénéficiaires. Cela illustre aussi l'incidence négative des retards de paiement, liés aux dysfonctionnements ou aux difficultés financières des filières cotonnières, sur le bilan économique des producteurs cotonniers, qui doivent alors recourir à des crédits tardifs.

\subsubsection{Un recours aux crédits de trésorerie presque généralisé}

Bien que le nombre de crédits de trésorerie ait dépassé le nombre de producteurs suivis dans l'étude, nous avons observé une minorité de 27 producteurs suivis (5\%) qui n'y a pas recouru (Tab. 2). Ceux qui ont recouru à ces crédits se répartissaient à peu près à égalité entre un seul crédit et plusieurs. Le nombre limité des producteurs n'ayant pas eu recours au crédit n'empêche pas l'application des modèles d'analyse quantitative, mais la faible taille de l'échantillon réduit la puissance des tests statistiques (c'est-à-dire la capacité à atteindre le seuil de significativité statistique). Mais si la significativité statistique est néanmoins observée, c'est que les effets correspondants sont importants. 
Tableau 2. Crédits et rentabilité cotonnière selon le nombre de crédits de trésorerie.

Table 2. Credit and profitability of cotton growing according to the number of credits.

\begin{tabular}{|c|c|c|c|c|c|}
\hline 1 & Nombre d'exploitations & 27 & 282 & 228 & \\
\hline 2 & Crédits contractés, CFA/ha & & & & \\
\hline 3 & Crédit/remboursement des intrants & $112225^{\mathrm{a}}$ & $91406^{\mathrm{c}}$ & $96667^{\mathrm{b}}$ & 0,001 \\
\hline 4 & Engrais & 45568 & 44122 & 41862 & 0,059 \\
\hline 7 & Dont insecticides & $61010^{\mathrm{a}}$ & $44309^{\mathrm{c}}$ & $50357^{\mathrm{b}}$ & 0,000 \\
\hline 8 & Crédit et remboursement du crédit de trésorerie & & & & \\
\hline 9 & Montant du crédit de trésorerie & & 45499 & $123138^{* * *}$ & $<0,0001$ \\
\hline 10 & Remboursement du crédit de trésorerie & & 85587 & $219806^{* * *}$ & $<0,0001$ \\
\hline 11 & Montant moyen d'un crédit de trésorerie & & 45499 & 55468 & 0,160 \\
\hline 15 & Marge après remboursement des intrants & 191159 & 165640 & 162671 & 0,442 \\
\hline 16 & Marge après remboursement des crédits intrants et de trésorerie & $191159^{\mathrm{a}}$ & $80052^{\mathrm{a}}$ & $-57135^{\mathrm{b}}$ & $<0,0001$ \\
\hline
\end{tabular}

Valeur de $p$ : test de Fisher; les moyennes associées à des lettres différentes sont significativement différentes au seuil de $5 \%$ dans les comparaisons 2 à 2 par le test de Newman-Keuls. ${ }^{* * *}$ : significatif au seuil de $99,9 \%$.

En dépit de cette limitation statistique, les résultats montrent que les producteurs sans crédit de trésorerie se distinguaient par un niveau de crédit intrants (Tab. 2, ligne 3), et donc potentiellement un niveau d'utilisation des intrants, supérieur à celui des producteurs qui ont dû y recourir. Cette différence provenait essentiellement de l'utilisation des insecticides (ligne 7), pour induire un rendement arithmétiquement plus élevé (ligne 13). Pour les producteurs ayant pris plus d'un crédit, le montant du crédit obtenu a été près de trois fois celui des producteurs à crédit unique (ligne 9), tout comme le montant à rembourser (ligne 10), alors que le montant moyen d'un crédit acquis n'a pas été significativement différent (ligne 11).

\subsubsection{Crédit de trésorerie, élément principal de remboursement}

Ramenée à l'hectare de coton cultivé, la valeur des crédits de trésorerie a été inférieure à celle du crédit intrants, du moins pour les producteurs n'ayant recouru qu'à un seul crédit de trésorerie. Par contre, en remboursement, compte tenu du niveau élevé des taux d'intérêt, la valeur a été presque égale à celle des crédits intrants (Tab. 2, lignes 3, 9 et 10) pour les producteurs ayant eu recours à un seul crédit de trésorerie, et de plus du double pour les producteurs ayant contracté de multiples crédits de trésorerie.

Pour l'ensemble des producteurs étudiés, la valeur du remboursement des crédits de trésorerie $(105$ millions de F CFA, Tab. 1) a été supérieure à celle des intrants fournis à crédits (97,3 millions de F CFA, valeur non présentée dans les tableaux), même en incluant les producteurs qui n'avaient pas recouru au crédit de trésorerie. Autrement dit, les intrants fournis à crédit n'ont pas été la principale source de ponction dans le revenu des paysans lors de la commercialisation du coton-graine.

Le manque de puissance des tests statistiques ne permet pas d'indiquer que la capacité d'une minorité de producteurs à ne recourir qu'à un seul crédit de trésorerie (plutôt qu'à plusieurs) procéderait clairement de différences dans les caractéristiques du chef d'exploitation, de sa famille, de son exploitation ou de sa culture cotonnière (Tab. 3). Les effets observés correspondent à ce qui était attendu selon les signes des paramètres (Tab. 4) mais seulement quelques-uns de ceux-là sont statistiquement significatifs. Le recours à un seul crédit de trésorerie (au lieu de plusieurs) est favorisé par le niveau de formation/éducation (grâce à l'alphabétisation fonctionnelle), qui permet probablement une meilleure appréciation de la rentabilité. Il en est de même des deux facteurs réduisant le besoin en main-d'œuvre temporaire que sont la disponibilité de jeunes pour travailler dans les champs et le recours aux herbicides. La significativité statistique de ces deux facteurs confirme que le crédit de trésorerie sert, au moins en partie, à payer la main-d'œuvre temporaire.

Le fait d'avoir plusieurs épouses a pour effet de pousser à prendre plusieurs crédits de trésorerie. Les raisons peuvent être diverses mais notre étude ne les a pas abordées et nous ne pouvons donc faire que des hypothèses. Dans les exploitations polygames, les femmes peuvent être moins disponibles pour travailler dans les champs de coton de leur mari, soit parce qu'elles sont plus occupées par les soins aux jeunes enfants, soit parce qu'elles peuvent se consacrer davantage à leurs propres champs. 
Tableau 3. Régression logistique sur le recours des producteurs au crédit de trésorerie.

Table 3. Logistic regression on farmers' use of cash credit.

\begin{tabular}{lc}
\hline Variables explicatives & Variance dépendante : recours à un seul crédit (vs plusieurs) \\
\cline { 2 - 2 } Caractéristiques de l'exploitation & Coefficient $b$ (déviation standard) \\
Âge du chef d'exploitation & \\
Nombre d'années à l'école du chef d'exploitation & $0,088(0,080)$ \\
Avoir bénéficié d'une alphabétisation fonctionnelle & $-0,064(0,068)$ \\
Nombre d'épouses & $0,181^{* *}(0,068)$ \\
Nombre de jeunes participants aux travaux des champs & $-0,147^{*}(0,064)$ \\
Nombre d'ouvriers agricoles & $0,154^{*}(0,063)$ \\
Superficie totale cultivée (ha) & $0,073(0,079)$ \\
Pratique la culture attelée & $-0,135(0,093)$ \\
Pratiques dans la culture du coton & $-0,063(0,062)$ \\
Nombre d'année de culture du coton & \\
Utilisation de l'herbicide & $0,082(0,081)$ \\
& $0,230^{* * *}(0,056)$ \\
Kh Log (vraisemblance) & \\
Pr $>$ Khi 2 & \\
\hline
\end{tabular}

${ }^{*},{ }^{* *},{ }^{* * *}:$ significatif aux seuils de 95,99 et $99,9 \%$.

Tableau 4. Effets attendus des variables explicatives du recours à un seul crédit de trésorerie (plutôt qu'à plusieurs).

Table. Anticipated effects of factors explaining the use of one cash credit (rather than several).

\begin{tabular}{lll}
\hline & \multicolumn{2}{l}{ Recours à un seul crédit de trésorerie vs plusieurs } \\
\cline { 2 - 3 } & Signe & Raison \\
\hline Caractéristiques de l'exploitation & & \\
Âge du chef d'exploitation & + & Expérience dans la culture \\
Nombre d'années à l'école & 0 & Peu discriminant entre les producteurs \\
A bénéficié d'alphabétisation & + & Meilleure connaissance en gestion \\
Nombre d'épouses & - & Disponibilité en travail réduite par l'âge des épouses ayant des enfants en bas âge \\
Nombre de jeunes au champ & + & Disponibilité en travail renforcée \\
Nombre d'ouvriers agricoles & 0 & Effet non perceptible par le faible nombre d'exploitations ayant des ouvriers \\
Superficie totale cultivée (ha) & - & Besoin en travail accru \\
Pratique la culture attelée & + & Peu discriminant car pratique très courante \\
Pratiques dans la culture du coton & & Meilleure maîtrise technique \\
Nombre d'années dans la culture & + & Besoin en travail réduit \\
Utilisation de l'herbicide & + &
\end{tabular}

Le signe + ou - indique l'effet d'un facteur en faveur (ou en défaveur) du recours à un seul crédit plutôt qu'à plusieurs. Par exemple, pour le cas de la variable âge, le signe + indique qu'on s'attend à ce que l'expérience associée à l'âge amène les producteurs à se limiter à un seul crédit, plutôt que d'en prendre plusieurs au risque de perdre toute rentabilité.

\subsubsection{Rentabilité faible et trappe de pauvreté}

Entre les producteurs ayant recouru ou pas au crédit de trésorerie, le ratio extrant/intrant et la marge après remboursement des intrants ne sont pas significativement différents (Tab. 5). Par contre, la valeur de la production après remboursement des crédits intrants et de trésorerie a été en moyenne très faible $(18721 \mathrm{FCFA}$ ou $28,5 € / \mathrm{ha})$ pour les producteurs ayant recouru au crédit de trésorerie, et très nettement inférieure à ceux qui n'y ont pas eu recours. Pour les producteurs ayant eu recours au crédit de trésorerie, le remboursement de ce dernier a absorbé pratiquement en totalité le revenu qui restait après le remboursement des intrants. Autrement dit, le recours au crédit de trésorerie conduit à amputer fortement le revenu du paysan, au profit de ses créanciers.

Le recours au crédit de trésorerie, dans les conditions observées, pénalise la rentabilité de la culture cotonnière. La distribution des producteurs selon le niveau atteint de MARICHI montre que l'absence de recours au crédit de 
Tableau 5. Performance comparée des producteurs recourant ou pas au crédit de trésorerie. Table 5. Comparative performance of farmers differentiated by their use of cash credit.

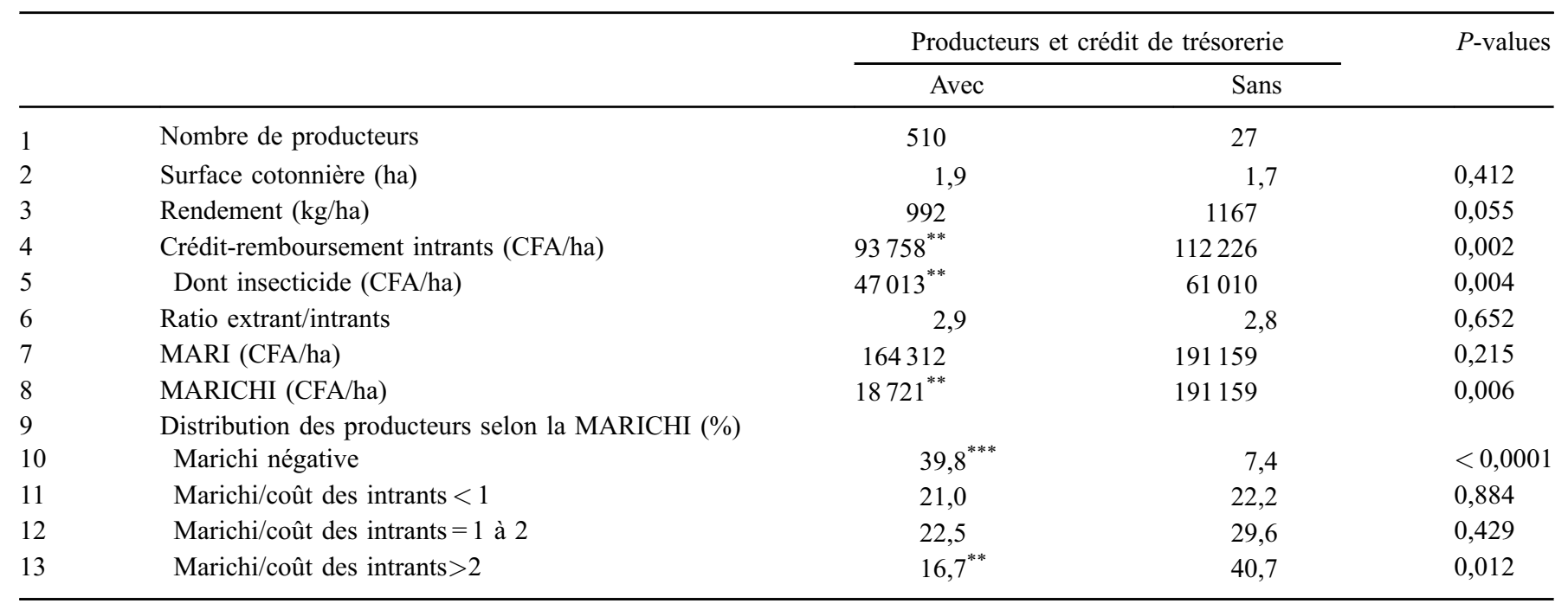

MARI : marge après remboursement des intrants ; MARICHI : marge après remboursement des intrants et hors intrants (trésorerie). ${ }^{*}{ }^{* *},{ }^{* * *}$ : significatif aux seuils de 95, 99 et 99,9\%.

trésorerie a plus fréquemment permis aux producteurs concernés de disposer d'un taux plus élevé de rentabilité (ratio MARICHI/ intrants supérieur à 2). À l'opposé, les producteurs recourant au crédit de trésorerie ont plus souvent souffert d'un revenu négatif après remboursement de ce crédit et du crédit intrant (cas de $40 \%$ de ces producteurs), près de six fois plus fréquemment que les producteurs n'ayant pas pris de crédit de trésorerie. On peut supposer que ces producteurs à marge négative ont dû engager d'autres sources de revenu que le coton (comme la vente sur pied du soja ou des noix de cajou) pour rembourser le crédit de trésorerie ou ont dû négocier un rééchelonnement de ce crédit, s'engageant ainsi dans la trappe de pauvreté.

Ce sont les conditions d'acquisition du crédit de trésorerie, avec des taux d'intérêt usuraires imposés par les acteurs informels (107,3\% en début de campagne et $202,2 \%$ en fin de campagne, contre $14,9 \%$ pour les acteurs formels), qui sont responsables de l'ampleur de l'impact négatif sur la rentabilité. $\mathrm{La}$ fréquence du recours combiné aux acteurs formels et informels n'a pas permis de calculer quels auraient été les effets si les acteurs formels avaient été les seuls à opérer, mais la différence entre les taux d'intérêt effectifs au moment du remboursement en donne cependant une idée.

\section{Conclusion}

Notre étude, réalisée dans le département des Collines au centre du Bénin, est la première à appréhender les modalités du crédit de trésorerie obtenu de manière informelle par les producteurs de coton. L'étude a pu être menée en profitant d'une initiative d'octroi de crédit formel de trésorerie, qui a attiré les opérateurs informels et a permis de révéler leurs pratiques.

En situation d'insuffisance de crédit formel de trésorerie, le recours au crédit informel a été quasi généralisé, malgré des conditions usuraires extrêmes. Les taux d'intérêt appliqués dépassent $100 \%$ sur une base annuelle, et même $200 \%$ pour les crédits alloués en fin de campagne.
Dans les conditions observées d'usure du crédit informel de trésorerie, le remboursement de ce type de crédit a été le principal élément de ponction dans le revenu du coton, dépassant le coût des intrants acquis à crédit. Le coût de ce crédit est tel qu'il absorbe une grande partie de la marge après le remboursement des intrants. Il en résulte que les producteurs concernés ont cultivé le coton essentiellement pour le profit des usuriers, dont la place dans la filière est jusqu'à maintenant occultée.

Bien que l'initiative d'octroi de crédits de trésorerie formels n'ait pas permis de faire face aux besoins des agriculteurs, les conditions d'accès au crédit informel mises en évidence soulignent l'importance cruciale d'avoir un système de crédit formel. Un taux d'intérêt bien plus faible, un huitième de celui appliqué par les usuriers, permet aux producteurs de conserver un revenu de leur culture de coton. Mais le crédit formel doit être octroyé de manière plus efficace et être ajusté en termes de calendrier et de volume pour pouvoir répondre aux besoins des producteurs, surtout quand, comme c'est fréquent, les sociétés cotonnières ont du retard pour collecter et surtout payer le coton.

L'étude a porté sur le Bénin, sur une région particulière du pays et dans un contexte particulier, il convient d'aborder le sujet du crédit de trésorerie des producteurs dans d'autres régions et pays. C'est ainsi qu'on pourra mieux cerner dans quelle mesure les opérateurs de crédit informels ont pu tirer profit des politiques cotonnières des pays concernés, souvent exécutées avec appui extérieur.

\section{Références}

Adams J, Brunner HP, Raymond F. 2003. Interactions of informal and formal agents in South Asian Rural Credit Markets. Review of Development Economics 7(3): 431-444.

Barslund M, Tarp F, 2008. Formal and informal rural credit in four provinces of Vietnam. Journal of Development Studies 44(4): 485-503. 
Bell C. 1990. Interactions between institutional and informal credit agencies in rural India. The World Bank economic review 4(3): 297-327.

Defo T. 2005. L'impact des circuits financiers informels et semiformels dans la mobilisation de l'épargne et le financement du développement : cas de l'Afrique francophone. African Development Review 9(1): 234-270. DOI: 10.1787/ 010624746507.

Eboh EC. 2000. Rural informal savings and credit associations as risk managers and the lessons for the design and execution of rural credit schemes in Nigeria. African Development Review 12(2): 233-262. DOI: $10.1111 / 1467-8268.00025$.

Kaino T. 2006. Rural credit markets in Myanmar: a study of formal and non-formal lenders. Asian Journal of Agriculture and Development 4(1): 1-15.

Kpadé P. 2011. Adaptation de la coordination et nouvelles contradictions entre acteurs du système coton au Bénin face à la libéralisation économique. Thèse de Doctorat. Dijon (France): Université de Bourgogne, $364 \mathrm{p}$.

Lele U, van de Walle N, Gbetibouo M. 1989. Cotton in Africa: an analysis of differences in performances. Washington: MADIA The World Bank, 37 p.

Posani B. 2009. Crisis in the countryside: farmers' suicide and the political economy of agrarian distress in India. Development
Studies Institute, Working paper Series No. 09-95. London, $52 \mathrm{p}$.

Poulton C, Gibbon P, Hanyani-Mlambo B, Kydd J, Maro W, Nylandsted Larsen M, et al. 2004. Competition and coordination in liberalized African cotton market systems. World Development 23 (3): 519-536.

Quartey P, Udry C, Al-Hassan S, Seshie H. 2012. Agricultural financing and credit constraints: the role of middlemen in marketing and credit outcomes in Ghana. Institute of Statistical, Social and Economic Research Working paper No. 12/0160. London: International Growth Center, $32 \mathrm{p}$.

Rosenberg R, Gonzalez A, Narain S. 2009. The new moneylenders: are the poor being exploited by high microcredit interest rates? Consultative group to assist the poor (CGAP) occasional paper No. 15. Washington DC, $28 \mathrm{p}$.

Sadanandan A. 2014. Political economy of suicide: financial reforms, credit crunches and farmer suicides in India. The Journal of Developing Areas 48(4): 287-307.

Thériault V, Tschirley DL. 2014. How institutions mediate the impact of cash cropping on food crop intensification: an application to cotton in Sub-Saharan Africa. World Development 64: 298-310. DOI: 10.1016/j.worlddev.2014.06.014.

Visvanathan S. 1998. The sadness of cotton. Economic and Political Weekly 33(7): 323-324.

Citation de l'article : Sossou KB, Fok M. 2019. Crédit de trésorerie des producteurs de coton au centre du Bénin: modalités et conséquences. Cah. Agric. 28: 14. 